\title{
Utilidad de la evaluación por otorrinolaringología en atención primaria de salud: Evaluación piloto
}

\author{
Usefulness of otolaryngology evaluation in primary health care: Pilot evaluation
}

Felipe Cardemil $\mathbf{M}^{1}$, Laura Hernández $\mathbf{R}^{1}$, Luis Cabezas $\mathbf{C}^{2}$, Cristina Sade $\mathbf{B}^{3}$, Luis Cabezas $\mathrm{L}^{1}$.

\begin{abstract}
RESUMEN
Introducción: En Atención Primaria de Salud (APS), las consultas por causas otorrinolaringológicas alcanzan hasta el 40\%-50\%. De éstos, muchos son enviados para evaluación al nivel secundario de salud.

Objetivo: Evaluar la utilidad de una atención de otorrinolaringología en APS para tamizar el número de interconsultas al especialista. Explorar estrategias que permitan replicar la experiencia.

Material y método: Se consideran las consultas de otorrinolaringología realizadas en dos escenarios diferentes, donde se evaluaron pacientes que se encontraban a la espera de atención formal por el nivel secundario.

Resultados: Se evaluaron 201 pacientes. El 60,9\% fueron de sexo femenino y el $39,1 \%$ masculino. La mediana de tiempo de espera para la derivación fue de 20 meses, con un rango intercuartil de 8-29 meses. El tiempo máximo de espera de interconsulta fue de 6 años (73 meses). Del total de interconsultas, 71,7\% fueron dadas de alta con tratamiento según la causa, mientras que sólo en el restante 28,3\% de las interconsultas se consideró necesario mantener la interconsulta al nivel secundario.

Conclusión: Los resultados de la presente evaluación permiten plantear la utilidad de una evaluación otorrinolaringológica en APS. Esto permitiría reducir el flujo de derivaciones y listas de espera hacia el sector secundario, descongestionar el sistema, priorizar las derivaciones más pertinentes, crear canales de comunicación expeditos entre los niveles de atención, contribuir a la capacitación continua en ambos equipos y niveles.
\end{abstract}

Palabras clave: Otorrinolaringología, atención primaria en salud, interconsulta.

\begin{abstract}
Introduction: In primary health care, medical appointments for otorhinolaryngological causes reach up to $40 \%-50 \%$. Of these cases, many are referred for the evaluation of the tertiary level of health.
\end{abstract}

1 Departamento de Otorrinolaringología, Clínica Las Condes y Facultad de Medicina, Universidad de Chile.

2 Servicio de Otorrinolaringología, Hospital Carlos Van Buren.

3 Psiquiatra, Servicio de Salud Metropolitano Sur.

Recibido el 25 de abril, 2017. Aceptado el 7 de mayo, 2017. 
Aim: Assess the usefulness of an otorhinolaryngology evaluation at the primary health care level to screen the number of referrals to specialist. Explore strategies to replicate the experience.

Material and method: Attention by otorhinolaryngologist is considered in two different scenarios, where patients who were waiting for formal care by the secondary level were evaluated.

Results: 201 patients were evaluated. $60.9 \%$ of the patients were females and $39.1 \%$ were males. The median waiting time for referral was 20 months, with an interquartile range of 8 - 29 months. The maximum waiting time for consultation was 6 years (73 months). Of the total number of consultations, $71.7 \%$ were discharged with treatment according to the cause, while only the remaining $28.3 \%$ of the consultations were considered necessary to maintain consultation at the tertiary level.

Conclusion: The results of this evaluation allow us to propose the usefulness of an otorhinolaryngological evaluation in primary health care. This would reduce the flow of referrals and waiting lists to the secondary sector, decongest the system, prioritize the most relevant referrals, create expedited channels of communication between levels of care, contribute to continuous training in both teams and levels.

Key words: Otolaryngology, Primary Health Care, Referral.

\section{INTRODUCCIÓN}

La atención primaria en salud (APS) es la asistencia esencial, puesta al alcance de todos los individuos y familias de la comunidad, constituyendo el primer nivel de contacto de éstos con el sistema nacional de salud ${ }^{1}$. El sistema público chileno atiende aproximadamente al $80 \%$ de la población, por lo que el acceso a la atención médica es cada vez más difícil, ocasionando listas de espera tanto de atención médica, generadas en parte por interconsultas realizadas hacia el nivel secundario y terciario, como de atención quirúrgica².

Se estima que las consultas por causas otorrinolaringológicas alcanzan entre el $40 \%$ y $50 \%$ en la atención primaria ${ }^{2,3}$. En Chile, entre las consultas del área respiratoria, se ha descrito que el $46 \%$ corresponden a causas de las vías respiratorias altas ${ }^{4}$. Los estudios indican que constituyen entonces la tercera especialidad más común a la que los médicos de atención primaria realizan interconsultas. Muchos de estos casos son derivados al nivel secundario o terciario de salud para la evaluación por un especialista, pese a que algunas patologías podrían considerarse susceptibles de ser resueltas a nivel primario de contarse con las herramientas diagnósticas adecuadas. Esta circunstancia favorece la saturación de los servicios de la especialidad y retarda la disponibilidad de atención especializada o quirúrgica en los casos que lo ameritan 5 .

\section{OBJETIVO}

Evaluar la utilidad de una evaluación otorrinolaringológica a nivel de APS para tamizar el número de interconsultas que se mantienen para recibir la atención por especialista en nivel secundario 0 terciario.

Explorar modalidades que permitan replicar la experiencia, y proponer hacerla sostenible en el tiempo.

\section{MATERIAL Y MÉTODO}

Se realizó un estudio de corte transversal en el que se evaluaron pacientes derivados a ORL, que se encontraban a la espera de la atención en la red formal y que habían consultado en la APS en dos escenarios diferentes: un consultorio de San Bernardo (policlínico "Rosita Benveniste"), y pacientes de la localidad de Chañaral que fueron atendidos en operativos médicos durante el año 2015. En ambos escenarios se consideraron los registros de las consultas del nivel primario durante los años anteriores y que, siendo derivados 
al nivel secundario, no habían sido aún evaluados (interconsultas sin resolver). Se incluyeron todos los pacientes con evaluación por la especialidad pendiente. Se identificó en cada paciente el diagnóstico que motivó la interconsulta, datos epidemiológicos, y el manejo recibido.

\section{RESULTADOS}

El total de personas atendidos fue 201, distribuidos en 87 pacientes de la comunidad de San Bernardo y 114 pacientes de la comunidad Chañaral, alcanzando $122(60,9 \%)$ sujetos femeninos y $79(39,1 \%)$ sujetos masculinos (Figura 1).

La edad media fue 33,3 años. La mediana de tiempo de espera para la derivación al otorrinolaringólogo fue de 20 meses (rango 8-29 meses). El tiempo máximo de espera para la interconsulta fue de 6 años (73 meses).

El diagnóstico más frecuente fue rinitis alérgica $(27,3 \%)$, seguido de patología adenoamigdalina $(23,9 \%)$. Los diagnósticos obtenidos se resumen en la Tabla 1.

De total de interconsultas, $144(71,7 \%)$ fueron dadas de alta con tratamiento según la causa y en 57 casos $(28,3 \%)$ se consideró necesario mantener la interconsulta al nivel secundario (Figura 2).

\section{DISCUSIÓN}

Estos resultados coinciden con algunos estudios similares, en cuanto a las causas de referencia

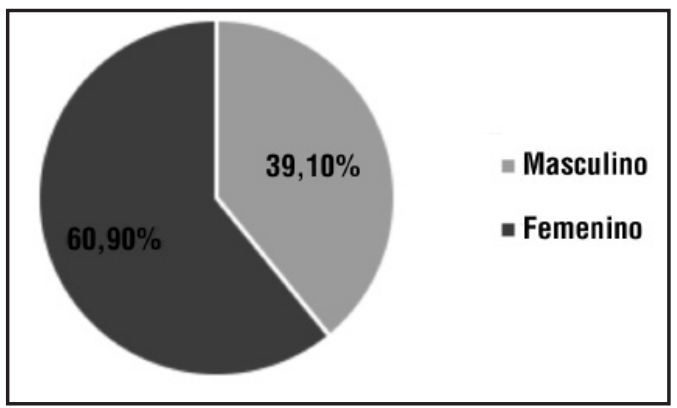

Figura 1. Distribución por sexo de los pacientes atendidos en el Policlínico de Otorrinolaringología de las comunidades San Bernardo y Chañaral (2015). desde el nivel primario, que fueron en primer lugar rinitis alérgica y patología adenoamigdalina, seguidas de causas otológicas como tapón de cerumen, hipoacusia y vértigo. Según Chávez ${ }^{5}$, en un estudio realizado en un hospital de segundo nivel en Ciudad de México para identificar las causas y proporción de pacientes referidos a la consulta de otorrinolaringología, las causas más frecuentes de derivación al especialista fueron síntomas nasales $(43 \%)$ y faríngeos $(38 \%)$, describiendo que $36 \%$ de los pacientes podrían haber sido tratados en el primer nivel de atención. Por otro lado, una serie chilena del año 2006 indicaba que la causa más frecuente de interconsulta de otorrinolaringología serían problemas relacionados con la hipoacusia $(32 \%)$, seguidos de las patologías de adenoamígdalas (32\%), oído medio y nariz ${ }^{6}$.

Un estudio de la India ${ }^{7}$ evaluó de manera prospectiva durante un período de tres años los diag-

Tabla 1. Distribución de diagnósticos frecuentes en el Policlínico de Otorrinolaringología de las comunidades San Bernardo y Chañaral (2015)

\begin{tabular}{|lrr|}
\hline Diagnóstico & $n$ & $\%$ \\
\hline Rinitis alérgica & 55 & 27,3 \\
Patología adenoamigdalina & 48 & 23,9 \\
Tapón de cerumen & 41 & 20,4 \\
Hipoacusia & 32 & 15,9 \\
Síndrome vestibular & 16 & 7,9 \\
Otros & 9 & 4,5 \\
\hline
\end{tabular}

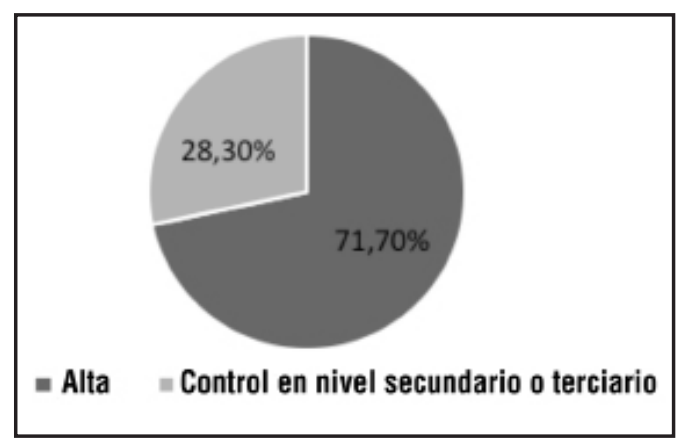

Figura 2. Porcentajes de interconsultas que fueron resueltas con la intervención y que fueron derivadas al nivel secundario en el Policlínico de Otorrinolaringología realizado en las comunidades San Bernardo y Chañaral (2015). 
nósticos de consulta en otorrinolaringología en atención primaria y secundaria, describiendo los trastornos más comunes, su causa y tratamiento. Los síntomas otológicos fueron en este caso los más frecuentes $(60 \%)$, predominando en niños la otitis media aguda (27\%) y presbiacusia (19\%) en la población adulta. Encontraron rinitis aguda en el $50 \%$, rinitis alérgica en el $35 \%$ y sinusitis recurrente en $8 \%$. Amigdalitis aguda recurrente fue el diagnóstico faríngeo más frecuente en niños (49\%) seguido de faringitis y laringitis aguda/crónica que correspondió al $19 \%$ de la muestra.

El tiempo de espera medio para la evaluación por el especialista en el nivel superior fue de 20 meses en esta serie, lo que debería alertar en cuanto a la dificultad de acceso a consultas especializadas y costos en el sistema de salud por retraso diagnóstico. La Asociación Médica Canadiense realizó un estudio ${ }^{8}$ buscando evaluar el proceso de derivación desde el sistema primario de salud hacia los especialistas en otorrinolaringología, la cual fue la segunda especialidad más difícil para gestionar derivaciones. Los médicos de atención primaria, en general, mencionaban descontento en cuanto a los tiempos de espera para consulta de otorrinolaringología.

La evaluación tardía por otorrinolaringología está asociada a un incremento en los costos de atención en salud. Así se demostró en un análisis retrospectivo de pacientes diagnosticados con patología laríngea ${ }^{9}$, en el quedó de manifiesto que se reducen los costos en salud al disminuir el tiempo entre la primera consulta de atención primaria y la primera consulta de otorrinolaringología, y la demora en el diagnóstico de cáncer laríngeo tiene un impacto adverso en la supervivencia de los pacientes.

En ocasiones, existe la necesidad de que patologías de la esfera otorrinolaringológica, después de ser confirmadas y tratadas por un especialista, continúen su seguimiento en la atención primaria y esto va de la mano con la tendencia actual hacia el manejo de enfermedades cada vez más complejas en la comunidad para evitar la saturación del servicio secundario y terciario $^{10}$. Esto hace que sea imprescindible la existencia de canales de comunicación expedita y continua. Addison y $\mathrm{col}^{10}$ evaluaron la comunicación efectiva entre los médicos otorrinolaringólogos y los de atención primaria, concluyendo que estos últimos prefieren los formatos de contrarreferencia estructurados, con títulos y subtítulos señalando los puntos clave, por razones de facilidad de comprensión, utilidad y legibilidad, minimizando el riesgo de que el lector pase por alto información importante acerca del diagnóstico, tratamiento y manejo del paciente. Según Gitterman y col ${ }^{11}$, la comunicación no sólo es importante para mejorar la calidad de atención, sino también un medio de educación de los médicos de atención primaria. Particularmente, en el manejo de la enfermedad de los senos paranasales, la atención integral y coordinada permite que las interconsultas se realicen temprano en el transcurso de pacientes complicados.

En el escenario de inequidad de muchos países en vías de desarrollo, es necesario mencionar que la asociación demostrada entre bajo estatus socioeconómico y el incremento en las atenciones de salud no se observa tan claramente en otorrinolaringología como en otras especialidades ${ }^{12}$. Esto permite plantear incluso que, a pesar que esta muestra estaba constituida por consultantes de comunas con población con mayor dificultad para acceso a atenciones de salud, esto no tendría un impacto en los resultados encontrados en el presente estudio. Es decir, esta situación podría ser similar en la mayoría de las otras comunas del país.

Independientemente de la distribución de las causas, los resultados de este estudio permiten plantear que un porcentaje alto de los pacientes que consultan a la atención primaria en salud por problemas relacionados con patologías otorrinolaringológicas, podrían ser manejados en el mismo nivel sanitario, sin necesidad de continuar su derivación al nivel secundario o terciario. De esta forma, se haría un uso más eficiente de los servicios de salud, filtrando el número de interconsultas con el especialista y mejorando el acceso a la atención médica, los tiempos de espera y el retraso en el manejo de patologías complejas 0 de resolución quirúrgica que efectivamente necesiten del nivel terciario. Considerando esto, se proponen cuatro posibles alternativas que podrían ser planteadas a los administradores en salud para intentar mejorar esto: 1) Fortalecer la instrucción de pregrado en la materia junto con fomentar actividades de educación médica continua en otorrinolaringología para médicos de APS que sean realizadas en el 
nivel primario de atención; 2) Contratar otorrinolaringólogos con jornadas parciales en APS; 3) Crear sistemas de telemedicina que permitan reuniones periódicas entre médicos otorrinolaringólogos de la red de salud y médicos de la APS, donde resolver dudas respecto a casos puntuales; 4) Fomentar la participación de residentes de otorrinolaringología en actividades en consultorios de APS. La primera alternativa es clave, y en ese sentido, organizaciones como las sociedades científicas (SOCHIORL), universidades, y hospitales 0 clínicas, son fundamentales para canalizar estas iniciativas que promuevan la capacitación continua de los médicos de APS en otorrinolaringología; estas actividades deberían mantenerse y estimularse. La segunda alternativa parecería más difícil de realizar, ya que implicaría que médicos otorrinolaringólogos dedicaran parte de su jornada en la APS, así como costos adicionales en un contexto de un sistema de atención primaria que necesita solucionar otros déficits en primer lugar. Sin embargo, hay experiencias nacionales en otras especialidades que evalúan de manera positiva el impacto a nivel de reducción de interconsultas y conformidad en ambos equipos de atención ${ }^{13}$. La tercera alternativa es una solución adecuada, en el sentido que la telemedicina es una herramienta que ha mostrado buenos resultados en otras especialidades $^{14}$, incluso en el contexto chileno ${ }^{15}$. En este sentido, habría que destinar tiempo de médicos especialistas de la red pública, los que muchas veces se encuentran con sus jornadas completas con actividades asistenciales, asociado a situaciones de listas de espera que requieren ser solucionadas. Es por esto, que el invertir en capacitación y comunicación entre los sectores involucrados contribuiría a la educación médica de los profesionales de la APS, así como a disminuir secundariamente el número interconsultas. La cuarta alternativa parece adecuada, considerando que los residentes de especialidad reciben un entrenamiento formal y realizan policlínico de atención ambulatoria en los hospitales donde se desempeñan, por lo que estarían capacitados para tamizar pacientes en el nivel primario que hayan sido derivados por los médicos de la APS hacia el nivel secundario; es decir, no deberían dedicarse a realizar un policlínico en la APS para pacientes con consultas nuevas (no evaluados previamente por un médico en APS), sino evaluar en la APS a pacientes que se encuentran en lista de espera, de manera de brindar atención a los casos menos complejos que se puedan resolver (por ejemplo, un cuadro de tapón de cerumen 0 rinitis alérgica), así como también realizar una derivación más expedita en un caso que requiera una evaluación más urgente (por ejemplo, un fumador con disfonía y adenopatías cervicales). Incluyendo esta actividad en un contexto docente que sea compatible con las actividades realizadas en la formación. En cualquier caso, el análisis de estas alternativas depende de la interacción de todos los actores relevantes, lo que incluye al Ministerio de Salud, sociedades científicas, universidades, y la comunidad en general, teniendo en consideración que se puede optimizar el sistema de interconsulta desde APS hacia el nivel secundario o terciario, y así facilitar una comunicación más expedita y colaborativa entre los equipos.

\section{CONCLUSIONES}

Los médicos de atención primaria deben contar con los recursos, herramientas y conocimientos necesarios para realizar el diagnóstico y tratamiento, o de ser requerido, gestionar la derivación oportuna de pacientes con enfermedades otorrinolaringológicas. Con una mayor capacidad resolutiva del nivel primario de atención, es posible una disminución de la morbimortalidad de estos pacientes, al igual que en los costos del sistema sanitario relacionados con el retraso en el diagnóstico y tratamiento.

En este estudio se propone incorporar la evaluación por un médico con formación en otorrinolaringología en atención primaria. Ésta resultaría beneficiosa no sólo para disminuir la cantidad de las interconsultas dirigidas a la especialidad a nivel secundario y terciario, al disminuir además tiempos de espera de consultas en este nivel, si no que se posibilitaría la creación de un espacio común de aprendizaje y comprensión de la patología, su afrontamiento y resolución y contribuiría a la formación continua de médicos y equipos clínicos. La intervención podría plantearse dentro el contexto de consulta directa, en conjunto con el médico tratante, en formato de consultoría clínica o como telemedicina. Otra alternativa sería que los 
residentes de otorrinolaringología asistieran a la APS, como parte de las actividades de su formación, a realizar evaluaciones en conjunto con los médicos de la APS, a pacientes con interconsulta

\section{BIBLIOGRAFÍA}

1. Zurro M.A., Sola Jodar, G. (2011) Atención primaria de salud y atención familiar y comunitaria. Madrid: Elsevier.

2. Díaz L, Dávila J, Rodríguez $V$, Añaños $D$. Frecuencia de diagnósticos de la especialidad de otorrinolaringología en el consultorio de medicina general en un centro de salud. Rev Med Hered 2003; 14(4): 163-6.

3. Mir N, Trilla A, Quintó LL, Molinero M, Asenjo M. ¿Qué papel tiene la otorrinolaringología en la asistencia primaria? Un análisis de variación en áreas concretas. Acta Otorrinolaringol Esp 2002; 53: 495-501.

4. Olmos C, Mancilla P, Martínez L, Astudillo P. Epidemiologia de las consultas respiratorias de adultos en Santiago de Chile desde 2003 a 2008. Rev Med Chile 2015; 143: 30-8.

5. Chávez G. Causas de referencia a la consulta de otorrinolaringología, en un hospital de segundo nivel. Rev Hosp Jua Mex 2009; 76(4): 190-3.

6. Der C, Salín M, Iñiguez M, Valenzuela P. Realidad de la derivación a otorrinolaringología desde atención primaria. Rev Otorrinolaringol Cir Cabeza y Cuello 2006; 66: 95-102.

7. Emerson LP, Job A, Abraham V. A Model for Provision of ENT Health Care Service at Primary and Secondary Hospital Level in a Developing Country. BioMed Research International 2013, Volume 2013, Article ID 562643, 5 pages.

8. Scott JR, Wong E, Sowerby LJ. Evaluating the referral preferences and consultation requests of primary care physicians with otolaryngology- en lista de espera. Esto permitiría reducir el flujo de interconsultas y listas de espera hacia el sector terciario, descongestionar el sistema y priorizar las derivaciones más pertinentes.

head and neck surgery. $J$ of Otolaryngol - Head \& Neck Surg 2015; 44: 57.

9. Cohen S, Kim J, Roy N, Courey M. Delayed otolaryngology referral for voice disorders increases health care cost. American Journal of Medicine 2015; 128(4): 11-8.

10. Addison AB, Watts S, Fleming J. Effective communication between ENT and primary care- a survey of outpatient correspondence. Clin Otolaryngol 2015; 40: 191-6.

11. GitTerman B. Frontline evaluation and treatment of patients with sinus disease: how otolaryngologist can halp primary care providers manage patients with sinusitis. Curr Opin Otolaryngol Head Neck Surg 2013; 21: 58-60.

12. Lous J, FriIs K, Vinding AL, Fonager K. Social marginalization reduces use of ENT physicians in primary care. Int J Pediatr Otorhinolaryngol 2012; 76(3): 370-3.

13. Tejada L, Sepúlveda R. Consultoría Psiquiátrica en Atención Primaria en el contexto del Modelo de Psiquiatría basado en la Comunidad: La experiencia Lo Espejo 2007. Servicio de Psiquiatría, Complejo Asistencial Barros Luco. Sociedad Chilena de Salud Mental. www. schilesaludmental.cl/2008_julio11/Taller_6.ppt.

14. John C. Fortney, Jefrerey M. Pyne, Eric E. Turner, Kellee M. Farris, Tre M. Normoyle, Marc D. Avery, Donald M. HILTY \& Jürgen Unützer. Telepsychiatry integration of mental health services into rural primary care settings. International Review of Psychiatry 2015; 27: 6, 525-39.

15. Castro A, Larraín A, Fritsch R, Rojas G. Telepsiquiatría: una revisión sistemática cualitativa. Rev Med Chile 2012; 140: 789-96. 